\title{
The Rate of Cerebral Blood Flow in the Clinic Of Traumatology and Orthopedics
}

\author{
Vladimir Alexeevich Schurov* \\ Research Center for Rehabilitating Traumatology and Orthopedics, Russia
}

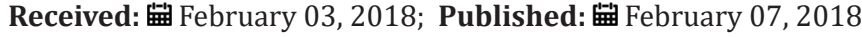

*Corresponding author: Vladimir Alexeevich Schurov, Ilizarov Research Center for Rehabilitating Traumatology and Orthopedics, Kurgan, Russia.

\section{Editorial}

Assessed by ultrasound Doppler blood flow velocity (BFV) in middle cerebral arteries (MCA) reflects the state of the body of patients. A study of representative samples of healthy people of different age showed that the BFV has been steadily declining with increasing age. In healthy people, the BFV is reduced from $123 \mathrm{~cm} /$ $\mathrm{s}$ at a rate $0,707 \mathrm{~cm} / \mathrm{s}$ per year. The decline of $60 \%$ from the original values leads to disruption ofthecapacityof the centers in the brain and infact the entire body.The value of BFV in the MCA, as well as the life expectancy of women is higher than men's, increased by $6 \%$ in chronic inflammatory diseases of the joints and reduced by $8 \%$ in the treatment of fractures of the limbs. However, these changes in patients almost affect the life expectancy of the patients. In patients with congenital violation of the natural growth of the limbs from childhood BFV in the MCA falls to $30 \%$. In subsequent years, the pace of decline is slowing.

The reason for the decline in BFV - in the necessary preservation of auto regulation of cerebral blood flow required for the functioning of his centers. The smaller the source the absolute value of BFV, the more it changes in the functional muscle samples. As a measure of the ability of vessels to respond adequately to changes in the level of afferentation, we used the magnitude of the changes in the index of BFV at the MCA in functional muscle samples (the compression elastic expander of each of the brushes). Thisscaleisanaverageof $22 \% \pm 1.6$ andshouldnotexceed $25 \%$.The scope exceeds this threshold in patients in the period of operative limb lengthening and in the treatment of bone fractures of extremities in patients in the first 2 weeks after trauma and in patients older than 50 years old in subsequent periods of treatment. In such periods, to limit the motive mode of patients. BFV in the MCA, increased in patients after traumatic brain injury and stroke at 6 and 13 months begins to decline.

The reduction reaches $40-60 \%$. The output of the "negative phase" indicator in patients with traumatic brain injury comes only after 5 years. The source healthy people, the magnitude of the increment BFV in the MCA contra lateral side of the brain when trauma is directly proportional to the amount of damaged body (collarbone and 21\%, shoulder - 34\%, hip - 59\%). The more caudal is damaged organ, the younger the leveling of the indicators of BFV to MCA on the contra lateral and ipsilateral sides of the brain (TBI -65 years old, shoulder -63 , thigh and lower leg $-43-37$ years). The definition of BFV in the MCA and changes in muscle load samples is highly informative in the treatment patients in the clinic of traumatology and orthopedics.
To Submit Your Article Click Here: This work is licensed under Creative Commons Attribution 4.0 License

DOI: $10.32474 /$ RRHOAJ.2018.01.000103

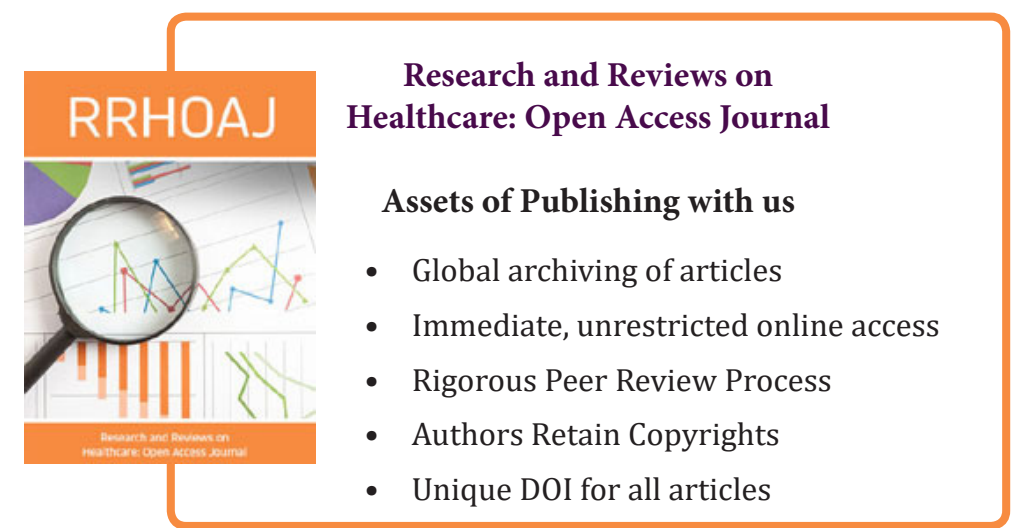

